\title{
The Global Convergence of a New Spectral Conjugate Gradient Method
}

\author{
W.Q. Zeng
}

The School of Computer Science of Guangdong Polytechnic Normal University

Guangzhou, Guangdong, China

zwq6381508@163.com

\author{
H.L. Liu \\ The School of Computer Science of Guangdong Polytechnic \\ Normal University \\ Guangzhou, Guangdong, China \\ lklhl@sina.com
}

\begin{abstract}
In this pager, we propose a new spectral Conjugate Gradient method to solve unconstrained optimization problems. It has the following properties: (1) the new method satisfies the sufficient descent condition with any line searches condition; (2) this method possesses inherent properties when $\theta_{k} \geq 0$ (3) under the strong Wolfe line search the method is globally convergent. Preliminary numerical results show that method is very efficient.
\end{abstract}

Keywords-sufficient decent; conjugate gradient method; line search; global convergence

\section{INTRODUCTION}

For solving the nonlinear unconstrained optimization problem

$$
\min _{x \in R^{n}} f(x)
$$

Where $f: R^{n} \rightarrow R$ is a continuously differentiable and its gradient is available, bound from below. Generally, we use the iterative method to solve (1), and the iterative formulas is given by

$$
x_{k+1}=x_{k}+\alpha_{k} d_{k}
$$

Where the $x_{k}$ is current iterative, $\alpha_{k}$ is a positive scalar and named the step-size, determined by some line search, $d_{k}$ is the search direction defined by

$$
d_{k}=\left\{\begin{array}{l}
-g_{k}, k=1 \\
-g_{k}+\beta_{k} d_{k-1}, k>1
\end{array}\right.
$$

Where $g_{k}=\nabla f\left(x_{k}\right)$ is the gradient of $\mathrm{f}(\mathrm{x})$ at the $x_{k}$ and $\beta_{k}$ is a scalar which determined different conjugate gradient methods [1, 2].The line search in the conjugate gradient algorithms often is based on the general Wolfe condition $[3,4]$ :

$$
\begin{aligned}
& f\left(x_{k}+\alpha_{k} d_{k}\right) \leq f\left(x_{k}\right)+\delta \alpha_{k} g_{k}{ }^{T} d_{k} \\
& g\left(x_{k}+\alpha_{k} d_{k}\right)^{T} d_{k} \geq \sigma g_{k}{ }^{T} d_{k}
\end{aligned}
$$

Where $0<\delta<\sigma<1$. There are many well known formulas for $\beta_{k}$, such as the Fletcher-Reeves(FR) [5],
Polar-Ribiere-Polyak(PRP) [6, 7] .Hestenes-Stiefe(HS) [8], and Conjugate Descent(CD)[9], Dai-Yuan(DY)[10]Quite recently, Birgin and Martinez [11] propose a spectral conjugate gradient method by combining conjugate gradient and spectral gradient method .Unfortunately, the spectral conjugate gradient method cannot guarantee to generate descent direction. So base on the FR formula, Zhang et al. [12] modified the FR conjugate gradient method.

$$
d_{k}=\left\{\begin{array}{l}
-g_{k}, k=1 \\
-\theta_{k} g_{k}+\beta_{k}^{F R} d_{k-1}, k>1
\end{array}\right.
$$

Where $\beta_{k}=\frac{\left\|g_{k}\right\|^{2}}{\left\|g_{k-1}\right\|^{2}}, \quad \theta_{k}=\frac{d_{k}{ }^{T}\left(g_{k}-g_{k-1}\right)}{\left\|g_{k-1}\right\|^{2}}$

And they proved the global convergence of the modified spectral FR method with the mild conditions. And then Huabin Jiang and Songhai Deng [13] propose a spectral conjugate gradient method about PRP method, they proof the global convergence under the Armijo line search, they proof when the exact line search is used, when $\theta_{k}=1$.

\section{NEW SPECTRAL CONJUGATE GRADIENT METHOD}

Consider the general spectral conjugate gradient method (2) and

$$
d_{k}=\left\{\begin{array}{l}
-g_{k}, k=1 \\
-\theta_{k} g_{k}+\beta_{k} d_{k-1}, k>1
\end{array}\right.
$$

Where $\alpha_{k}$ (2) is a step-size satisfy the Wolfe condition(4),(5)and $\theta_{k}$ is a coefficient, $\beta_{k}$ is a scalar which determined different spectral conjugate gradient methods .We assume that the search direction is descent direction for $\mathrm{k}-1$ steps, and we hope the conclusion also for k steps, so we have

$$
g_{k}^{T} d_{k}<0
$$

From the (7) and multiplying by $g_{k}{ }^{T}$, and from the (8) we have

$$
-\theta_{k}\left\|g_{k}\right\|^{2}+\beta_{k} g_{k}{ }^{T} d_{k-1}<0
$$


We observe the formula (9), let the form of $\theta_{k}$ to be $\theta_{K}=1+A_{K}$, and we assume $\beta_{k}>0$, we have

$$
\frac{\theta_{k}\left\|g_{k}\right\|^{2}}{\beta_{k}}>g_{k}{ }^{T} d_{k-1}
$$

From (5) we have

$$
\frac{\left\|g_{k}\right\|^{2}}{\beta_{k}}+\frac{A_{k}\left\|g_{k}\right\|^{2}}{\beta_{k}}=-g_{k-1}{ }^{T} d_{k-1}+g_{k}{ }^{T} d_{k-1}
$$

From our assume we known that $g_{k-1}{ }^{T} d_{k-1}<0$ and $\beta_{k}>0$, and from (11) we let

$$
\begin{aligned}
& \frac{\left\|g_{k}\right\|^{2}}{\beta_{k}}=-g_{k-1}{ }^{T} d_{k-1} \\
& \frac{A_{k}\left\|g_{k}\right\|^{2}}{\beta_{k}}=g_{k}{ }^{T} d_{k-1}
\end{aligned}
$$

From the (12), (13) we know that

$$
\begin{gathered}
\theta_{k}=1-\frac{g_{k}{ }^{T} d_{k-1}}{g_{k-1}{ }^{T} d_{k-1}} \\
\beta_{k}=-\frac{\left\|g_{k}\right\|^{2}}{g_{k-1}{ }^{T} d_{k-1}} \\
\text { III. ALGORITHM AND LEMMAS }
\end{gathered}
$$

In order to establish the global convergence of the proposed method, we need the assumption on objective function, which have been used often in the literature to analyze the global convergence of nonlinear conjugate gradient with inexact line search.

\section{Assumption 3.1}

(1) The level set $\Omega=\left\{x \mid f(x) \leq f\left(x_{1}\right)\right\}$ is bounded, where $\mathrm{x}_{1}$ is the starting point.

(2) In some neighborhood $\mathrm{N}$ of $\Omega$, the objective function is continuously differentiable, and its gradient is Lipchitz continuous ,so there exists a constant $\mathrm{L}>0$ such that

$$
\|g(x)-g(y)\| \leq L\|x-y\|, \forall x, y \in N
$$

\section{Algorithm 3.2}

Step 1. Data: $x_{1} \in R^{n}, \varepsilon \geq 0$.Set $d_{1}=-g_{1}$, if $\left\|g_{1}\right\| \leq \varepsilon$, then stop, otherwise go to Step 2;

Step 2.Computer $\alpha_{k}$ by some line search

Step3.Let $x_{k+1}=x_{k}+\alpha_{k} d_{k}, g_{k+1}=g(k+1)$,if $\left\|g_{k+1}\right\| \leq \varepsilon$, then stop, otherwise go to the Step 4.

Step 4.Computer $\beta_{k+1}$ by (15), and generate $d_{k+1}$ by (7)

Step 5.Set $\mathrm{k}=\mathrm{k}+1$, go to Step 2

Lemma 3.3. Consider any method (2), (7), where(14),(15) and the step-size $\alpha_{k}$ be determined by the any line search, then

$$
g_{k}^{T} d_{k}<0
$$

Proof: From the (7) and multi plying by $g_{k}{ }^{T}$,the parameter determined by (71), (72), we have

$$
g_{k}{ }^{T} d_{k}=-\left(1-\frac{g_{k}{ }^{T} d_{k-1}}{g_{k-1}{ }^{T} d_{k-1}}\right)\left\|g_{k}\right\|^{2}-\frac{\left\|g_{k}\right\|^{2}}{g_{k-1}{ }^{T} d_{k-1}} g_{k}{ }^{T} d_{k-1}=-\left\|g_{k}\right\|^{2}
$$

From the above, we obtain that the conclusion

And from the the equation (15) and (17) we have

$$
\beta_{k}=\frac{g_{k}{ }^{T} d_{k}}{g_{k-1}{ }^{T} d_{k-1}}
$$

Lemma 3.4 Support that assumption (16) holds, Consider any method (2), (7), where(14),(15) and the step-size $\alpha_{k}$ be determined by the Wolfe line search, then

$$
\sum_{k \geq 1} \frac{\left(g_{k}{ }^{T} d_{k}\right)^{2}}{\left\|d_{k}\right\|^{2}}<+\infty
$$

The above lemma (19) often called Zoutendijk condition, is used to prove the global convergence properties of nonlinear conjugate gradient methods. It was originally given by Zoutendijk.

\section{GLOBAL CONVERGENCE PROPERTY}

Theorem 20 .Suppose that Assumption (16) holds, Consider any method (2), (7), where(14),(15) and the step-size $\alpha_{k}$ be determined by the Strong Wolfe line search, then

$$
\lim _{k \rightarrow+\infty} \text { in }\left\|g_{k}\right\|=0
$$

Proof: Support by contradiction that there exists a positive constant $\gamma>0$, such that

$$
\left\|g_{k}\right\| \geq \gamma
$$

From (7), we have $d_{k}+\theta_{k} g_{k}=\beta_{k} d_{k-1}$, and by squaring it, we get

$$
\left\|d_{k}\right\|^{2}=\beta_{k}{ }^{2}\left\|d_{k-1}\right\|^{2}-2 \theta_{k} g_{k}{ }^{T} d_{k}-\theta_{k}{ }^{2}\left\|g_{k}\right\|^{2}
$$

From the above equation (18)and dividing by $\left(g_{k}{ }^{T} d_{k}\right)^{2}$ we have

$$
\begin{aligned}
& \frac{\left\|d_{k}\right\|^{2}}{\left(g_{k}{ }^{T} d_{k}\right)^{2}}=\frac{\left\|d_{k-1}\right\|^{2}}{\left(g_{k-1}{ }^{T} d_{k-1}\right)^{2}}-2 \frac{\theta_{k}}{g_{k}{ }^{T} d_{k}}-\theta_{k}{ }^{2} \frac{\left\|g_{k}\right\|^{2}}{\left(g_{k}{ }^{T} d_{k}\right)^{2}} \\
= & \frac{\left\|d_{k-1}\right\|^{2}}{\left(g_{k-1}{ }^{T} d_{k-1}\right)^{2}}-\left(\frac{\theta_{k} g_{k}}{g_{k}{ }^{T} d_{k}}+\frac{1}{g_{k}}\right)^{2}+\frac{1}{\left\|g_{k}\right\|^{2}} \\
\leq & \frac{\left\|d_{k-1}\right\|^{2}}{\left(g_{k-1}{ }^{T} d_{k-1}\right)^{2}}+\frac{1}{\left\|g_{k}\right\|^{2}}
\end{aligned}
$$


Using recursively and $\left\|d_{1}\right\|^{2}=-g_{1}{ }^{T} d_{1}=\left\|g_{1}\right\|^{2}$, we have

$$
\frac{\left\|d_{k}\right\|^{2}}{\left(g_{k}{ }^{T} d_{k}\right)} \leq \sum_{i=1}^{k} \frac{1}{\left\|g_{i}\right\|^{2}}
$$

From (21) and (24), we have

$$
\frac{\left(g_{k}{ }^{T} d_{k}\right)^{2}}{\left\|d_{k}\right\|^{2}} \geq \frac{\gamma^{2}}{k}
$$

Which indicates

$$
\sum_{k \geq 1} \frac{\left(g_{k}{ }^{T} d_{k}\right)^{2}}{\left\|d_{k}\right\|^{2}}=+\infty
$$

This contradicts the Zoutendijk condition (19).Therefore the conclusion (20) holds.

\section{THE INHERENT PROPERTY OF THE METHOD AND ITS APPLICATIONS}

We will introduce the inherent nature of the method under the any line search condition and we define

$$
q_{k}=\frac{\left\|d_{k}\right\|^{2}}{\left(g_{k}{ }^{T} d_{k}\right)^{2}}
$$

And

$$
r_{k}=-\frac{g_{k}{ }^{T} d_{k}}{\theta_{k}\left\|g_{k}\right\|^{2}}
$$

We use (28), (29) change the equation (23), we have

$$
q_{k}=q_{k-1}+\frac{1}{\left\|g_{k}\right\|^{2}} \cdot \frac{2}{r_{k}}-\frac{1}{\left\|g_{k}\right\|^{2}} \cdot \frac{1}{r_{k}^{2}}
$$

We assume exit positive scalar $\gamma$ and $\bar{\gamma}$ satisfy

$$
\gamma \leq\left\|g_{k}\right\| \leq \bar{\gamma}, \forall k \geq 1
$$

Lemma5.1 Consider the method (2) ,(7),(14),(15), $\theta_{k}>0$ and(16) if(31) holds, there exit positive constants $\delta_{1}, \delta_{2}, \delta_{3}$, such that relations

$$
\begin{gathered}
-g_{k}{ }^{T} d_{k} \geq \frac{\delta_{1}}{\sqrt{k}} \\
\left\|d_{k}\right\|^{2} \geq \frac{\delta_{2}}{\sqrt{k}} \\
r_{k} \geq \frac{\delta_{3}}{\sqrt{k}}
\end{gathered}
$$

Proof: Using (30) recursively, and noting that $d_{1}=-g_{1}$, and $q_{k} \geq 0$,we have

$$
\frac{1}{\left\|g_{k}\right\|^{2}}\left(-\frac{2}{r_{k}}+\frac{1}{r_{k}^{2}}\right) \leq \sum_{i=1}^{k-1} \frac{1}{\left\|g_{i}\right\|^{2}}\left(\frac{2}{r_{i}}-\frac{1}{r_{i}^{2}}\right)
$$

From (35) and (31) we have

$$
\frac{1}{r_{k}^{2}}-\frac{2}{r_{k}}-\frac{\bar{\gamma}}{\gamma}(k-1) \leq 0
$$

From (36) and $r_{k}>0$, we have

$$
\frac{1}{r_{k}} \leq 1+\sqrt{1+\frac{\bar{\gamma}^{2}}{\gamma^{2}}(k-1)} \leq 1+\frac{\bar{\gamma}}{\gamma} \sqrt{k} \leq \frac{2 \bar{\gamma}}{\gamma} \sqrt{k}
$$

From (37), we have

$$
r_{k} \geq \frac{\delta_{3}}{\sqrt{k}} \quad \delta_{3}=\frac{\gamma}{2 \bar{\gamma}}
$$

From (29), we have

$$
\begin{gathered}
-g_{k}{ }^{T} d_{k}=\left\|g_{k}\right\|^{2} r_{k} \theta_{k} \\
\left\|d_{k}\right\| \geq\left\|g_{k}\right\| r_{k} \theta_{k}
\end{gathered}
$$

From (39), (40), (38) and assume $\theta_{k} \geq \xi$,we have

$$
\begin{gathered}
-g_{k}{ }^{T} d_{k} \geq \frac{\delta_{1}}{\sqrt{k}} \\
\left\|d_{k}\right\|^{2} \geq \frac{\delta_{2}}{\sqrt{k}}
\end{gathered}
$$

And $\delta_{1}=\delta_{3} \gamma^{2} \xi, \delta_{2}=\delta_{3}^{2} \gamma^{2} \xi$

Now we will construct a new line search condition:

$$
f_{k}-f_{k-1} \geq c \min \left\{-g_{k}{ }^{T} d_{k},\left\|d_{k}\right\|^{2}, q_{k}{ }^{-1}\right\}
$$

Which $\mathrm{c}$ is a scalar and c>0, $q_{k}$ is from (5.1) and the assumption (16) holds. We can easy to proof

$$
f_{k}-f_{k-1} \geq c q_{k}^{-1}
$$

By the Wolfe line search condition and we can also proof

$$
f_{k}-f_{k+1} \geq c \min \left\{-g_{k}{ }^{T} d_{k}, q_{k}{ }^{-1}\right\}
$$

By the Armijo line search condition. So from the (44), we can know that it satisfy Wolfe line condition and Armijo line condition.

\section{NUMERICAL EXPERIMENTS}

From the derivation of the two parameter $\beta_{k}$ and $\theta_{k}$ we known that is very similar with the DY methods, and D.Y prove it global convergence under the Wolfe line search condition. The parameter $\beta_{k}$ in the new spectral conjugate gradient method is the same to the $\mathrm{CD}$ conjugate gradient method. FR is the famous conjugate gradient methods. So in this section, we compare the Spectral conjugate gradient method, donated the SP method, to FR method, CD method under the strong Wolfe line search with the given initial points and dimensions. The parameters are chosen as follows: $\delta=0.01, \sigma=0.1$. The problems we test are from [14], 
If $\left\|g_{k}\right\| \leq 10^{-6}$ is satisfied, we will stop the program. We will be also stopped the program if the number of iteration is more then 9999. All codes were written in FORTRAN90 and run on a PC with 2.00GHz CPU processor and $2.00 \mathrm{G}$ Memory and Windows XP operation system.

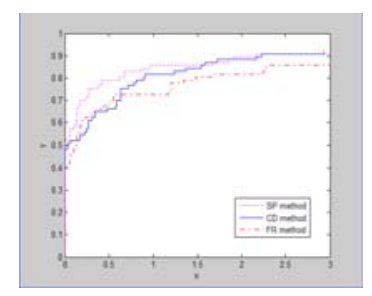

FIGURE I. PERFORMANCE PROFILES FOR THE NUMBER OF ITERATIONS.

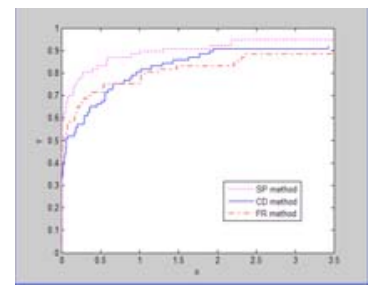

FIGURE II. PERFORMANCE PROFILES FOR THE NUMBER OF FUNCTION EVALUATIONS.

We adopt the performance profile by Dolan and More [15] to compare the SP method with FR method, and CD method. Figure 1-4 show the performance of the three methods relative to the number of iterations, the number of function evaluations, the number of the gradient evaluations and the CPU time.

Obviously, from the four figures show that SP method outperforms FR method, CD method for the given test problems in CPU time, the number of function evaluations, and the number of gradient evaluations, so the SP method is computationally efficient.

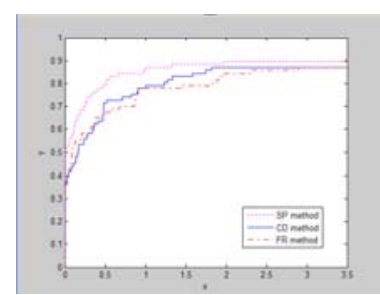

FIGURE III. PERFORMANCE PROFILES FOR THE NUMBER OF GRADIENT EVALUATIONS.

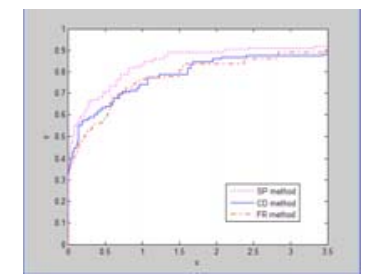

FIGURE IV. PERFORMANCE PROFILES FOR THE CPU TIME.

\section{REFERENCES}

[1] G.H.YU, Nonlinear self-scaling conjugate gradient methods for large-scale optimization [Ph.D. thesis], Sun Yat-Sen University, Guangzhou, China 2007

[2] G. Yuan and Z. Wei, New line search methods for unconstrained optimization .Journal of the Korean Statistical Society, vol.38, no.1, pp.29-39, 2009

[3] Wolfe, P., Convergence conditions for ascent methods, SIAM Rev., Vol. 11, pp.226-235, 1968

[4] Wolfe, P., Convergence conditions for ascent methods, (II ): some corrections. SIAM Review 13 (1971) 185-188

[5] Fletcher, R. and Reeves, C. M. "Function minimization by conjugate gradients,” The Computer Journal, vol. 7. pp. 149-154, 1964

[6] E. Polak and G. Ribiere, "Note sur la convergence de methods de direction conjugate,” vol. 3, no. 16, pp.35-43,1969.

[7] Polyak, B.T., The conjugate gradient method in extreme problems. USSR Comp. Meth. Meth. Phys. 9, 94-112(1969)

[8] M. R. Hestenes and E. stiefel, "Methods of conjugate gradients for solving linear systems," Journal of Research of the National of Standards, vol. 49, pp.409=436, 1952

[9] R, Fletcher, Practical methods of Optimization: Unconstrained Optimization, vol. John Wiley\&Sons, New York., NY, USA, 1987

[10] Dai, Y.H., Yuan, Y., A nonlinear conjugate gradient method with a strong global convergence property, SIAM. Math. Optim. 43, 87-101(2001)

[11] E. G. Birgin and J. M. Martinez, "A Spectral conjugate gradient method for unconstrained optimization,” Applied Mathematics and Computation, vol. 180, pp. 46-52, 2006

[12] L. Zhang, W. Zhou, and D. Li, “Global convergence of a modified Fletcher-Reeves conjugate gradient method with Armijo-type line search,” Numerische Mathematic, vol. 104, no.64, pp. 561-572, 2006

[13] Huabin Jiang, Songhai Deng, Xiaodong Zhang, and Zhang Wan, "Global Convergence of a Modified Spectral Conjugate Gradient Method,” Journal of Applied Mathematics, 2012

[14] J.J. More, B.S. Garbow and K.E. Hillstrom, Testing unconstrained optimization software, ACM Transactions on Mathematical Software $7(1981) 75-82$

[15] E.D. Dolan and J.J. More, "Benchmarking optimization software with performances profiles,” Mathematical Programming, vol. 91, no. 2, pp.201-213, 2002 